\title{
Background Independence: Lessons for Further Decades of Dispute
}

\author{
Trevor Teitel \\ New York University
}

October 1, 2018

Forthcoming in Studies in History and Philosophy of Modern Physics

\begin{abstract}
Background independence begins life as an informal property that a physical theory might have, often glossed as 'doesn't posit a fixed spacetime background'. Interest in trying to offer a precise account of background independence has been sparked by the pronouncements of several theorists working on quantum gravity that background independence embodies in some sense an essential discovery of the General Theory of Relativity, and a feature we should strive to carry forward to future physical theories. This paper has two goals. The first is to investigate what a world must be like in order to be truly described by a background independent theory given extant accounts of background independence. The second is to argue that there are no non-empirical reasons to be more confident in theories that satisfy extant accounts of background independence than in theories that don't. The paper concludes by drawing a general moral about a way in which focussing primarily on mathematical formulations of our physical theories can adversely affect debates in the metaphysics of physics.
\end{abstract}

\section{Introduction}

Background independence begins life as an informal property that a physical theory might have, often glossed as 'doesn't posit a fixed spacetime background'. Einstein's General Theory of Relativity (hereafter 'GR') is standardly taken as the paradigm example of a background independent theory. Interest in trying to offer a precise account of background independence has been sparked by the pronouncements of several theorists working on quantum gravity that background independence embodies in some sense an essential discovery of GR, and a feature that we should strive to carry forward to future physical theories. Some theorists then use this claim to argue against certain approaches to quantum gravity (most 
notably perturbative string theories), on the grounds that these approaches have to date been background dependent. ${ }^{1}$ There's a scarcity of empirical tests we can feasibly carry out that might help tease the different programs in quantum gravity apart, so getting clear on whether background independence can help in this regard is of significant interest in our search for a theory of quantum gravity and a final physical theory more generally.

There's now a sizable and growing literature amongst physicists and philosophers offering different mathematical accounts of background independence. ${ }^{2}$ This literature is largely concerned with technical issues about which mathematical formulations of various theories do or don't turn out background independent relative to each of these precise accounts. Although this investigation is one that must be done, we should be wary of getting embroiled in these mathematical questions and losing sight of why we're supposed to even be interested background independence in the first place.

This paper has two goals, both of which are aimed to remedy this issue. The first is to investigate the metaphysical question of what a world must be like in order to be truly described by a background independent theory given extant accounts of background independence, i.e. to investigate what non-mathematical significance the various mathematical properties that have been offered as accounts of background independence might have, which is a question that has surprisingly received little attention in the literature. My second goal is to ask whether we should in general be more confident that our world is in fact some such way than that it isn't, as some proponents of background independence have claimed. Notice that the two questions are intertwined: only once we get clear on the former metaphysical issue can we begin to evaluate the epistemological claim that we should in general be more confident in theories that satisfy extant accounts of background independence than in theories that don't. After all, properties of a physical theory that ought to make us more confident that the theory is true must pertain to what the theory says about the world, i.e.

\footnotetext{
${ }^{1}$ To get a feel for some of these pronouncements about background independence being a crucial innovation of GR see Rovelli $(1997,2001)$, Baez (2001), and Smolin $(2006 a, b)$, though be warned that several different properties are appealed to in the literature under the heading of 'background independence'. Also, the exact choice of words describing the alleged special status of background independence in GR varies, but all of the standard phrases convey a similar idea. The "essential discovery" locution I used in the main text is from Rovelli $(1997,209)$. Elsewhere Rovelli describes background independence as a "central discovery of GR" and a "discovery that is forever" $(2001,107,117-118)$. Baez $(2001,181)$ calls it "the great lesson of general relativity". And Smolin calls it "the most basic insight of Einstein's theory" (2006a, 198), "the chief lesson of general relativity" $(2006 \mathrm{~b}, 54)$, and says that it's "a basic discovery that cannot be reversed, so any further theory must incorporate it" (2006b, 239). It's easy to find many more analogous claims made on behalf of background independence throughout the philosophical literature on GR and quantum gravity.

${ }^{2}$ For a sampling see Earman (2006a,b), Rickles (2008), Belot (2011a), de Haro (2015), de Haro et al. (2016), Pooley (2017), and the works cited in footnote 1. For some helpful surveys of the literature see Norton (1993), Giulini (2007), Read (2016), and Pooley (2010, 2017). As I'll explain in section 2.1 below, work on general covariance is arguably continuous with work on background independence, hence my including Earman's analysis of "substantive general covariance" on this list (indeed the two articles by Pooley just cited describe the same properties but under these two different headings).
} 
its propositional content, not which mathematics we use to express the theory. (Note that throughout the paper my talk of being more or less confident in some theory should be understood in terms of being more or less confident that the theory is true, not merely that the theory is empirically adequate or has some other property.)

I need to clarify the second goal somewhat. Very broadly, there are two forms an argument to be more confident in background independent theories than background dependent ones might take. The first would be an empirical argument, which appeals to GR's background independence and its empirical successes when compared with earlier nonbackground independent theories. The second form of argument would point to some nonempirical (apriori) considerations in favor of background independence. Here I'll focus on the prospects of defending background independence using arguments of the second kind, which we'll see arguably must figure in any case for background independence that's to have a chance of succeeding. The upshot is largely negative: given this restriction, there's little to be said in favor of theories that satisfy extant accounts of background independence over those that don't.

You might immediately have the following worry: the most charitable reconstruction of what theorists who tout the importance of future physical theories being background independent have in mind will in large part appeal to the empirical successes of GR, so why care about whether these theorists have anything to say once we set this resource aside? I agree that the empirical successes of GR must figure prominently in the most compelling case for background independence. Nevertheless, we'll see in section 2.3 that our question-whether there are any non-empirical considerations that support background independence-arguably can't be separated from evaluating the empirical case for background independence, and is also an important question to investigate for independent reasons. Moreover, I already mentioned that my first goal here is to investigate what constraints extant mathematical accounts of background independence are used to impose on the nonmathematical world. This issue is relevant to those working on background independence irrespective of their views on how to argue for the importance of future physical theories being background independent. Finally, many issues we'll discuss turn on general topics in the foundations of spacetime theories and modal metaphysics, including questions of haecceitism and the significance of a physical theory's mathematical solutions with respect to what the theory says about the world (i.e. its propositional content). So much of our discussion should be of interest even setting aside the issue of background independence.

You might immediately also have another worry: everybody knows that it's hard to offer an apriori explanation of why various familiar non-empirical virtues that scientists use to adjudicate between rival theories should have any evidential relevance, so isn't my argument that there's no such explanation to be had for background independence just an 
instance of this more general problem facing everyone? This worry misses the mark. The crucial disanalogy is that whereas some might take an epistemic norm in favor of familiar non-empirical virtues (such as simplicity, unifying power, etc.) as a basic norm that can't be explained in other terms, I take it that nobody would take an analogous position about background independence, which is a term of art applicable only to physical theories. So the defender of the importance of future physical theories being background independent has an obligation to offer some explanation of why we should be more confident in background independent theories. And crucially, I'll argue that even granting that apriori there's an epistemic norm in favor of theories that enjoy traditional non-empirical virtues like simplicity, and so granting the proponent of background independence these additional resources she might use to justify her position, there's still no non-empirical reason to be more confident in background independent theories. Of course, if it turns out that those who argue against the evidential relevance of non-empirical virtues like simplicity are correct, then the prospects for proponents of background independence become even dimmer than I'll show them to be here, but I'll concede these resources in what follows.

Here's the plan. First, in section 2, I'll say some more to situate the issue of background independence, and then clarify the questions I'll focus on here and explain why they're important ones to investigate. Next, in section 3, I'll spell out three prominent accounts of background independence, in terms of diffeomorphism invariance, absolute objects, and a recent account due to Gordon Belot. In the rest of the paper (sections 4, 5, and 6), I'll investigate what worlds must be like in order to be truly described by theories satisfying these accounts, and also argue that none of these accounts vindicates the claim that there's non-empirical reason to be more confident in background independent theories than their alternatives. I'll conclude in section 7 by summing up my results and drawing a general moral for theorizing about background independence and the metaphysics of physics more broadly.

\section{Stage Setting}

\subsection{Preliminaries}

The 'what's special about General Relativity?' debate was conducted for much of the 20th century under the guise of a debate over whether any property can serve the role that Einstein once envisaged for general covariance (see Norton (1993) for a virtuoso survey of this history). Glossing over several historical details, the story goes as follows. Einstein once claimed that the distinctive feature of his GR was its equations being statable in any coordinate system without the need for additional correction terms, a property he called 'general covariance'. Soon afterwards, Kretschmann objected that even non-relativistic spacetime 
theories and Special Relativity can be given generally covariant formulations, and hence that general covariance doesn't serve to distinguish GR from earlier theories (this point is easy to see in hindsight given now familiar coordinate-free formulations of such theories). This "Kretschmann objection" engendered the ensuing search for a replacement for general covariance, i.e. a property of theories that would in fact distinguish GR from earlier theories. The topic of background independence is continuous with this older question. Those theorizing about the notion take themselves to be isolating a property of theories that's in some sense an essential feature of GR, and hence might serve the role Einstein once envisaged for general covariance.

Now of course there must be other constraints restricting what an adequate account of background independence might be besides distinguishing GR from earlier theories. For instance, the property of being consistent with experimental results about Mercury's orbit would on its own distinguish GR from Newtonian gravitational theories, though nobody would consider this property a candidate for the sort of "essential discovery" of GR embodied in background independence. This point becomes even more apparent when we consider other properties that would distinguish GR from earlier theories, such as being first discovered on Earth after 1912, being Bob Wald's favorite theory, being the theory described in the textbook closest to me while writing this sentence, and so on.

In practice the discussion proceeds via a familiar process of reflective equilibrium. Physicists and philosophers working on the topic take themselves to have some reasonably determinate judgments about which theories should or shouldn't turn out background independent, which they can then use to test accounts. The discussion can be further disciplined using the guiding principle that background independence should distinguish GR from earlier theories, and also the various informal glosses on the notion (e.g. 'doesn't posit a fixed spacetime background' and 'doesn't posit any non-dynamical fields'). None of these constraints is taken as non-negotiable; for instance, even the guiding principle to distinguish GR from earlier theories can be satisfied to various degrees, depending on whether one considers every formalization of the relevant theories or only some, general relativistic theories with any sort of matter fields or only certain paradigm general relativistic theories, and so on. Together these judgments and principles trade off against one another and help narrow down the space of candidate accounts of background independence.

Although this methodology can narrow down the space of options, I doubt it'll privilege any one account of background independence over the others given the inevitable indeterminacy in judgments about which theories should or shouldn't turn out background independent. More importantly, once we distinguish the various different accounts, the question of which one best coheres with the judgments of the relevant experts and the various guiding principles, and so which most deserves the honorific 'background independence', is 
largely a matter of terminology and predominantly of sociological interest. By contrast, the question of whether any of the proposed accounts fulfills the promise of providing a tool to help adjudicate between rival future physical theories goes beyond settling on terminology, and for my purposes it doesn't matter which of the accounts if any we deem deserving of the label 'background independence'. Certainly if any of the accounts is in fact a property that can help us adjudicate between rival theories then that would give us ample reason to be interested in which theories satisfy the property, irrespective of what we choose to call it.

\subsection{Non-Empirical Virtues}

As mentioned in the introduction, my second goal here concerns whether there's non-empirical reason to be more confident in theories that satisfy extant accounts of background independence than in theories that don't. It will be helpful to have some terminology for this topic, so let's adopt the following stipulative definition throughout:

A property of physical theories is a non-empirical virtue just in case given all pairs of rival theories such that the first theory satisfies the property but the second theory doesn't, we ceteris paribus ought to be more confident that the first theory is true than that the second theory is true, to some non-trivial degree, and solely for non-empirical reasons.

In this terminology, we're asking whether any extant accounts of background independence express properties that are non-empirical virtues. Moving forward I'll generally write as if we're asking whether extant accounts themselves are non-empirical virtues, which makes for an easier read. This is misleading, however, because generally the accounts are mathematical properties that on their own say nothing about the world. So officially my talk of whether some account of background independence is a non-empirical virtue should be understood as asking whether the constraint on the world that account is being used to express (i.e. the non-mathematical property the account is being used to represent) is a non-empirical virtue. I'll return to this contrast in the conclusion.

Three clarifications about the definition. First, by the "to some non-trivial degree" qualifier I mean that we must be more confident in the first theory than in the second to a sufficient degree such that if an account of background independence were to be a non-empirical virtue then that would justify the kinds of claims made on behalf of background independence as a tool for adjudicating between rival future physical theories (see the works cited in footnote 1 for some examples). I won't try to make the qualifier any more precise, but it's clear from these claims that being more confident by only a tiny amount that would be outweighed by most countervailing considerations won't be enough here. Second, I'll often omit the 'ceteris paribus' and 'to some non-trivial degree' qualifiers in what follows, 
though they should be understood as tacit throughout when I discuss which properties are non-empirical virtues. Finally, you'll notice that I use unqualified normative language like 'ought' and 'reason' when discussing non-empirical virtues. Such talk should always be understood in terms of epistemic normativity.

On this last point, let me stress that the definition ties non-empirical virtues to epistemically rational degrees of confidence. So challenging whether extant accounts of background independence are non-empirical virtues leaves open that background independence might serve other roles. For example, my arguments don't bear on the use of background independence as a heuristic to help us construct new physical theories that then plays no role when we're evaluating which theories are likely to be true.

\subsection{Why Care?}

Before turning to various extant accounts of background independence, let me list a few reasons to be interested in whether these accounts are non-empirical virtues.

First, I'm not sure there's a purely empirical case to be made that we generally ought to be more confident in future theories that are background independent. The empirical successes of a theory support the theory as a whole (setting aside theories with components that play no role in generating the theory's empirical predictions). As a result, these empirical successes on their own can't privilege some feature of the theory over the rest as one we should expect to appear in future physical theories. So arguably some non-empirical considerations are needed to motivate privileging background independence over the countless other properties of GR as a property we should be confident will be preserved in future physical theories given GR's empirical successes (again see footnote 1 for examples of such claims made on behalf of background independence). If that's right then any successful defense of the importance of background independence as a tool for adjudicating between rival future physical theories must involve the claim that background independence is a non-empirical virtue, which I'm challenging here.

Second, even setting the previous point aside, my conclusion that extant accounts of background independence aren't non-empirical virtues reveals exactly what must be done in order to argue that we ought to be generally more confident simpliciter in background independent theories. In particular, my conclusion shows that any successful attempt to justify the claims made on behalf of background independence as a tool for adjudicating between rival future physical theories must go via empirical considerations. Even if this is in fact the line of defense most proponents of background independence would be inclined to take in the first place, showing that this choice is required, and that no other line of defense can succeed, is an important result. It's also worth stressing that certainly if someone could show that an account of background independence is a non-empirical virtue then that would 
immediately settle the general question of whether there's any reason simpliciter to be more confident in background independent theories, which on its own makes the question worth investigating.

Third, some proponents of the importance of background independence, most notably Smolin (2006a), seem to claim that we have some apriori reasons to be more confident in background independent theories. In general in both Rovelli's and Smolin's writings (cited in footnote 1) it's hard to disentangle these kinds of considerations from an argument based on the empirical successes of GR; however it's clear that both place considerable importance on relationism about spacetime, the doctrine that spacetime is not fundamental, and at best somehow derivative from the spatiotemporal relations that obtain between matter. I won't attempt to reconstruct their reasoning here. ${ }^{3}$ What's uncontroversial is that both take the background independence of GR to make the theory amenable to relationism, and take this latter fact to support their claims about the importance of future physical theories being background independent. But plausibly any argument for the importance of background independence that stems from some alleged link between a theory being background independent and being amenable to relationism must go beyond the empirical successes of GR. Such an argument must instead appeal to some considerations supporting relationism, which will plausibly be largely apriori and so of the sort that could show background independence to be a non-empirical virtue. Thus arguably two of the most prominent advocates of the importance of background independence as a resource for adjudicating between future physical theories take their reasons to derive at least in part from background independence being a non-empirical virtue. My conclusion shows them to be mistaken in this regard. 4

Finally, as mentioned in the introduction, investigating whether any accounts of background independence are non-empirical virtues is only half of my aim here. My other goal is to get clear on what a world must be like in order to be truly described by a background independent theory given extant mathematical accounts of background independence. This metaphysical issue has surprisingly received little attention in the literature, and is relevant to those working on background independence irrespective of whether they're inclined to

\footnotetext{
${ }^{3}$ This isn't easy to do. After all, many take GR to vindicate substantivalism even given the hole argument. Moreover, it's generally thought that non-relativistic theories-paradigms of background dependence-offer the setting most hospitable to relationism. See Rickles (2008) for related skepticism about the relevance of background independence to the substantivalism vs. relationism debate.

${ }^{4}$ Even the phonebook seems to endorse apriori reasons to be more confident in background independent theories: when comparing GR to alternative gravitational theories, it says "Set aside ... the issue of agreement with experiment. Einstein's theory remains unique. Every other theory either introduces auxiliary gravitational fields ... or involves 'prior geometry,' or both. Thus, every other theory is more complicated conceptually than Einstein's theory" Misner et al. (1973, 429). However, they immediately add "Every other theory contains elements of complexity for which there is no experimental motivation," which suggests that their motivations here may not be purely apriori.
} 
care about which accounts, if any, express properties that are non-empirical virtues.

\section{Three Accounts of Background Independence}

In this section I'll outline three accounts of background independence, in terms of diffeomorphism invariance, lacking absolute objects, and a recent account due to Gordon Belot (2011a). I regard Belot's account as the most promising one formulated to date, so I'll outline it in more detail than the others, and it will be my main focus in what follows.

\subsection{Models and Field Theories}

We first need a framework for discussing the (always non-quantum) field theories that we'll be looking at in this paper. The physics and philosophy literature on such theories almost universally employs a model-theoretic framework, where a theory is expressed using its solution space, which is a class of mathematical models (sometimes called the theory's 'dynamically possible models'). In my view the use of this framework often has unfortunate consequences, despite its near-universal acceptance, because of its association with the socalled "semantic view of theories". ${ }^{5}$ However, for this paper I'll employ the standard modeltheoretic framework, though I'll flag places where it might lead us astray.

So every field theory we'll discuss is associated with a class of mathematical models, the space of solutions of the theory. In the theories we'll discuss such models will always have the form $\left\langle M, O_{1}, O_{2}, \ldots\right\rangle$, where $M$ is a four-dimensional differentiable manifold and the $O_{i}$ are geometrical objects (in particular we'll consider only theories where the $O_{i}$ are tensor fields on $M) .{ }^{6}$ For example, in this framework the space of solutions to a toy general relativistic Klein-Gordon theory is the class of all triples $\left\langle M, g_{a b}, \phi\right\rangle$-where $M$ is a fourdimensional differentiable manifold, $g_{a b}$ is a smooth Lorentzian metric field on $M$, and $\phi$ is a smooth scalar field on $M$-such that the objects in the triples satisfy both the Einstein equation (relating $g_{a b}$ and the stress-energy-momentum tensor for $\phi$ ) as well as the massless Klein-Gordon equation.

The heuristic you should have in mind is that the solution space of a theory is used to represent the space of nomic possibilities assuming that theory is true. These two spaces needn't be isomorphic, however, because of solutions that differ merely by a so-called 'gauge redundancy'. The rough idea here is that differences between the solutions of some theory are merely gauge differences when they arise only as an artifact of the mathematical formalism

\footnotetext{
${ }^{5}$ Roughly, the framework supports the myth that somehow by looking only at a mathematical object like a collection of models, independently of some story of how the models are being used to represent the world, one can extract a proposition imposing a constraint on what the world is like. I'll say more about this myth in the conclusion.

${ }^{6}$ This now-standard 'geometrical objects' terminology traces back to Anderson $(1967,14-16)$.
} 
we've happened to use to express the theory, rather than being mathematical differences we're using to represent differences that give rise to new nomic possibilities. ${ }^{7}$ The significance and extent of these merely gauge differences are hotly contested (and the term 'gauge' is used in several different ways). We'll get into some of the relevant issues later on, but for now the rough gloss will suffice.

Note that the accounts of background independence we're about to see were proposed in this mathematical setting of classical (non-quantum) field theories, given their focus on isolating some central property of GR. Of course there's a question of whether the mathematics employed by proposals for a final physical theory will be sufficiently similar to the differential geometry of the classical field theories we'll discuss so as to ensure that analogues of the various accounts can be written down in that mathematical formalism. If the answer turns out to be 'no' then attempts to carry forward some mathematical property of classical field theories to future physical theories must go indirectly, via whatever constraint on the non-mathematical world we're using that mathematical property to express (which future theories may or may not represent the world as satisfying). In my view, this provides considerable motivation for my first task here of distilling these non-mathematical properties from the extant mathematical accounts of background independence. In what follows I'll discuss the accounts of background independence only in the classical setting in which they were proposed, leaving for future work the question of how natural analogues of the accounts might be written down in other mathematics that might appear in current theories of quantum gravity and future physics more generally (see Belot (2011a, 2879) for brief discussion of this issue). ${ }^{8}$

\footnotetext{
${ }^{7} \mathrm{My}$ use of 'possibilities' rather than 'possible worlds' here is deliberate: these notions come apart given certain conceptions of possible worlds and the truth conditions for de re modality (for relevant discussion see Skow (2008)).

${ }^{8}$ An anonymous referee raises two worries about my claims in this paragraph:

Worry 1: The focus on classical field theories like GR renders the philosophical literature on background independence in general, and this paper in particular, disconnected from the relevant work on quantum gravity. However, remember that my focus (following the majority of the extant literature) is on background independence understood as preserving some "essential discovery" of GR (recall section 2.1), which explains the focus on classical field theories. The label 'background independence' is also sometimes used in the literature on quantum gravity in a manner that isn't meant to be preserving some feature of GR, but these uses aren't ones that the extant accounts of background independence I'm discussing are meant to capture. Moreover, the morals that I draw by reflecting on GR have more general significance; for example, the moral that we can't just focus on mathematics if we're interested in whether some property of a physical theory is a non-empirical virtue (I expand on this moral in the conclusion). These morals are neutral on the physical theory under discussion, and so can help us avoid conceptual confusions when we move to analogous debates in the context of future physical theories.

Worry 2: Talk of preserving some property in future physical theories should always be understood as requiring only that the future physical theory recover the property of the older theory via some limiting procedure, where the newer theory approaches the regime in which the older theory is applicable (compare the familiar non-relativistic limits of relativistic theories). I agree that in many contexts when we're asking whether some property is preserved we have such a procedure in mind, particularly when we're wondering whether some future physical theory can recover the predictions of the older theory. However, I think that often we don't have
} 


\subsection{Diffeomorphism Invariance}

Given two differentiable manifolds $M$ and $M^{\prime}$ (where $M^{\prime}$ may be identical to $M$ ), a diffeomorphism $d$ from $M$ to $M^{\prime}$, if one exists, induces a map from any tensor field $O$ defined on $M$ to a new tensor field defined on $M^{\prime}$ (the drag along of $O$ induced by $d$ ), which we'll label $d^{*} O{ }^{9}$ The way to think about these drag alongs is that $d^{*} O$ ascribes to a point $p$ of $M^{\prime}$ the same properties that $O$ ascribes to the point $d^{-1}(p)$ of $M$. Now consider a field theory $T$ (in the sense defined above) and a solution of that theory $\left\langle M, O_{1}, O_{2}, \ldots\right\rangle$. We know that given any four-dimensional differentiable manifold $M^{\prime}$, and any diffeomorphism $d$ from $M$ to $M^{\prime}$ (if one exists), we can define a new mathematical model $\left\langle M^{\prime}, d^{*} O_{1}, d^{*} O_{2}, \ldots\right\rangle$. We can now ask whether this new mathematical model is also a solution of $T$. Generalizing, we have the following definition:

Diffeomorphism Invariance: A field theory is diffeomorphism invariant iff, for any solution of the theory $\left\langle M, O_{1}, O_{2}, \ldots\right\rangle$, any four-dimensional differentiable manifold $M^{\prime}$, and any diffeomorphism $d$ from $M$ to $M^{\prime}$ (if one exists), $\left\langle M^{\prime}, d^{*} O_{1}, d^{*} O_{2}, \ldots\right\rangle$ is also a solution of the theory.

The standard lore says that general relativistic theories are diffeomorphism invariant whereas special relativistic and non-relativistic theories aren't, so it's unsurprising that some have put forward diffeomorphism invariance as a precise account of background independence. ${ }^{10}$

a limiting procedure in mind when asking whether some general property of a physical theory is preserved in future theories. For example, when we ask whether a future physical theory preserves determinism, Lorentz invariance, or time-translation invariance, we're generally asking whether these properties themselves are instantiated by the future theory, not whether the future theory recovers them in some limit where it approximates an older theory. And I think plausibly background independence, as those in the philosophical literature are understanding the notion, is exactly analogous to properties of the latter sort. Determinism provides a helpful analogy: the now-standard modal definition of determinism, prominent since Earman (1986), is applicable to any theory, so long as the theory has laws and hence allows us to investigate what's nomically possible according to the theory. As a result, we can inquire in the most straightforward sense as to whether this property of determinism is preserved in some future physical theory, without needing to worry about the mathematics in which the theory is couched or its limiting relations to older theories. The non-mathematical accounts of background independence I try to extract here (summarized in the conclusion) are also stated using nomic modality, and hence make background independence exactly analogous.

${ }^{9} \mathrm{~A}$ map $d$ is a diffeomorphism if it's a smooth $\left(C^{\infty}\right)$ bijection with a smooth inverse. See Wald (1984, 437438) for details on how different tensor fields (such as a Lorentzian metric) transform under the action of a diffeomorphism. Note the focus on diffeomorphisms throughout the literature is somewhat misleading; for many purposes to which diffeomorphisms are put (especially in discussions of the hole argument) the more general class of arbitrary permutations, smooth or not, would serve equally well (see Maudlin (1988, 84-85) and Melia (1999, 642-643)). Nevertheless, I'll generally focus on diffeomorphisms, which simplifies our discussion at various points and keeps it more in line with the existing literature.

${ }^{10}$ The falsity of this standard lore due to certain deviant special relativistic theories, and where this leaves accounts of background independence in terms of diffeomorphism invariance, have been much discussed (see Sorkin (2002), Earman (2006a,b), Giulini (2007), and Pooley (2017)). Here I'll set these issues aside, including different ways one might modify the notion of diffeomorphism invariance. The modified definitions fare no better than the one from the main text in terms of being non-empirical virtues, and discussing them and their motivations would be an unnecessary detour. 
We'll discuss later on what the significance of a theory satisfying this formal property might be.

\subsection{Absolute Objects}

The notion of an absolute object is due to Anderson (1967), and was later taken up and developed by Friedman (1983). Here we'll work with a coordinate-free definition now common in the literature. ${ }^{11}$ Given a field theory $T$, we can ask whether some geometrical object is the same up to global diffeomorphisms across all solutions of $T$, i.e. whether there's a geometrical object whose only degrees of freedom across solutions of $T$ can be induced by global diffeomorphisms. Formally, given a field theory $T$, we're looking for a type of geometrical object $O_{i}$ appearing in all solutions of $T$ and such that, for any two solutions $\left\langle M, O_{1}, O_{2}, \ldots\right\rangle$ and $\left\langle M^{\prime}, O_{1}^{\prime}, O_{2}^{\prime}, \ldots\right\rangle$ of $T$, there's some diffeomorphism $d$ from $M$ to $M^{\prime}$ such that $O_{i}^{\prime}=d^{*} O_{i}$. Call any such object an absolute object of the theory. With this definition in hand, it's a short step to the following account of background independence: a field theory is background independent just in case it contains no absolute objects.

It's not hard to see why this account of background independence might seem promising: the Lorentzian metric of general relativistic theories can possess degrees of freedom that go beyond what can be induced by global diffeomorphisms, unlike the metrics of paradigm non-relativistic or special relativistic theories. As a result, only the metrics of the latter theories turn out to be absolute objects. Again, we'll discuss later on what the significance of a theory lacking absolute objects might be.

\subsection{Belot's Account}

Notice that the two accounts of background independence we just saw are purely formal properties of theories, by which I mean properties we can test for by looking only at the mathematical models in a theory's solution space independently of some story about what those models are being used to represent. Belot contends from the get-go that we shouldn't expect background independence to be a property of this sort $(2011 \mathrm{a}, 2868)$, and this is why I take his account to be the most promising one currently available. I'll say more about this theme in the conclusion.

\footnotetext{
${ }^{11}$ See for instance Pitts (2006), Giulini (2007), and Pooley (2017), which also contain surveys of the standard issues facing accounts of background independence in terms of absolute objects. Note the definition I use is closer to Anderson's original proposal than to Friedman's, which uses local diffeomorphisms $(1983,57-60)$. Friedman's definition has the result that theories can allow the global topology of spacetime to vary across solutions yet nevertheless represent spacetime with an absolute object. In my view this result makes his definition less amenable to forging a link between being background independent and lacking absolute objects, given the various informal glosses on background independence we saw above. Those who disagree won't have trouble adapting the points I make in the main text about absolute objects to accommodate Friedman's definition, though we'll stick with the simpler definition throughout.
} 
The informal idea behind Belot's (2011a) account is to define a graded notion of background independence where "a theory is fully background independent if each physical possibility corresponds to a distinct spacetime geometry, and that it falls short of full background independence to the extent that this condition fails" (9). Picturesquely, for Belot the more background dependent a theory is, the more modal freedom matter has over and above the modal freedom of spacetime geometry across the theory's space of nomic possibilities; in one limiting case (full background independence) any differences across modal space correlate with differences in spacetime geometry, in the other limiting case (full background dependence), spacetime geometry has no modal freedom, i.e. remains fixed across the space of nomic possibilities, despite matter being free to move about. ${ }^{12}$

I'll present a somewhat simplified version of Belot's account (readers interested in the details should consult Belot (2011a, 2873-2879)). We just saw that Belot defines a theory's degree of background independence in terms of the extent to which the modal differences that the theory allows can't come apart from the modal differences in geometry that the theory allows. Belot labels the differences between a theory's solutions that represent modal differences the theory's physical degrees of freedom, and the differences between the theory's solutions that represent modal differences in geometry the theory's geometrical degrees of freedom (we'll define these terms precisely in a moment). Here's where the point above about background independence not being a purely formal property comes in: a class of mathematical models on its own is just some uninterpreted formalism, and so doesn't tell us anything about the world, let alone about the space of nomic possibilities according to some theory. We can extract such a story about modal differences only with an account of which elements of a theory's models are being used to represent spacetime geometry, which differences between these elements are being used to represent differences that generate new nomic possibilities, and also which differences between the theory's models more generally are being used to represent differences that generate new nomic possibilities (i.e. we need to decide which differences between models are "merely gauge" in the sense introduced above). So for Belot background independence is a property of more than just a class of mathematical models, but rather a property of a class of such models combined with the representational decisions that allow us to extract geometrical and physical degrees of freedom from the models. ${ }^{13}$

Starting with geometrical degrees of freedom, Belot (2011a, 2873) takes a "geometriza-

\footnotetext{
${ }^{12} \mathrm{~A}$ natural gloss here would be that full background independence requires everything to globally supervene with nomic necessity on spacetime geometry, and background independence fails to the extent that supervenience fails. However, 'supervenience', even in its global variety, is used in numerous ways, so I'll avoid the term (see Bennett \& McLaughlin (2014) for an overview of some options here).

${ }^{13}$ I'll use 'theory' to describe both a class of mathematical models (a solution space) and such a class together with some of these representational choices when no confusion will arise from this unfortunate ambiguity. Some may prefer to use the label 'theory' in only one of these senses, but I take this to just be a terminological issue.
} 
tion" of a theory to be a choice of (i) which elements of the theory's solutions represent geometrical structure and (ii) an equivalence relation on these elements determining which differences between elements represent differences that generate new nomic possibilities. For example, in general relativistic theories we naturally associate an uninterpreted solution $\left\langle M, g_{a b}, \ldots\right\rangle$ with a geometrization according to which the Lorentzian metric field $g_{a b}$ represents the geometrical structure of the manifold represented by $M$. To give an example of (ii), philosophers of physics often take the hole argument to show that we should endorse some form of anti-haecceitism, which I'll understand throughout as the doctrine that no metaphysical possibilities differ without differing qualitatively; I'll understand haecceitism as the negation of this doctrine. ${ }^{14}$ I don't think one can define the qualitative vs. non-qualitative distinction in independent terms. A rough gloss, though, is that a proposition is qualitative just in case it's not about any particular individuals (where all other propositions are haecceitistic or non-qualitative), and that possibilities differ qualitatively just in case they differ over the truth value of some qualitative proposition. Anti-haecceitists may want diffeomorphically related metrics to turn out equivalent according to the relation in (ii), assuming the standard representational convention that such metrics are used to represent geometries that differ only over which spacetime points play which qualitative geometric roles (which is a merely haecceitistic difference), and hence the metrics ascribe the same complete qualitative structure to spacetime.

Given a theory and a geometrization of that theory, we get a set of equivalence classes of mathematical objects, where the mathematical objects are those the geometrization says represent geometrical structure and the relevant equivalence relation is the one given by the geometrization. For Belot (2011a, 2874), any set of variables that parametrizes this set are the geometrical degrees of freedom of the theory-geometrization pair. For example, in standard general relativistic theories, the space of Lorentzian metrics that appear in some solution-even quotiented by the equivalence relation that holds between two such metrics when one is the drag along of the other induced by some diffeomorphism-is infinitedimensional. So given this definition standard general relativistic theories have infinitely many geometrical degrees of freedom. ${ }^{15}$

\footnotetext{
${ }^{14}$ For the hole argument see Earman \& Norton (1987) and the survey in Norton (2015). For some proponents of wedding substantivalism to anti-haecceitism see Field (1984), Brighouse (1994), Hoefer (1996), and Pooley (2006). Several debates go under the heading of 'haecceitism vs. anti-haecceitism'. Here I'm picking up on one standard usage (arguably dating back to Kaplan (1975) and more recently championed by Skow (2008)). Note that as I'm using the term, the debate doesn't directly concern the metaphysics of individuals or possible worlds, nor does it directly concern mathematical models and what they can be used to represent. Of course doctrines of these sorts may have implications for the debate as I've defined it.

${ }^{15}$ The formal details behind the claim of infinite-dimensionality in this paragraph, and what exactly the geometrical degrees of freedom (i.e. variables parametrizing the quotiented space of metrics) look like here, won't be important in what follows. Interested readers should consult Isenberg \& Marsden (1982), which discusses globally hyperbolic and spatially compact solutions, and Andersson (1987), which extends these results to solu-
} 
The account of physical degrees of freedom runs analogously. The difference is that here we look at the solution space of a theory, rather than the space of the elements in the theory's solutions that are being used to represent geometry, and quotient this solution space by the relation of gauge equivalence. The result is that solutions in different equivalence classes (i.e. those that differ in some respect that isn't merely gauge) differ in a way that we're understanding to represent a difference that generates new nomic possibilities. We'll impose the natural coherence constraint between geometric equivalence and gauge equivalence: one way for solutions to differ is over the elements (if any) they contain that the geometrization says represent spacetime geometry, so we'll require that if two solutions differ over such elements and the elements aren't geometrically equivalent then the solutions aren't gauge equivalent. ${ }^{16}$ Belot calls any set of variables that parameterizes the quotiented solution space (which represents the space of distinct nomic possibilities allowed by the theory) the physical degrees of freedom of the theory.

With these definitions in hand, Belot's account runs as follows (2011a, 2877):

Background Dependence (Belot): A field theory is background dependent if it has no geometrical degrees of freedom: for any two solutions, the elements of the first that represent geometrical structure are geometrically equivalent to the corresponding elements of the second.

Background Independence (Belot): A field theory is background independent if all of its physical degrees of freedom correspond to geometrical degrees of freedom: for any two solutions, the solutions are gauge equivalent if and only if the elements of the first that represent geometrical structure are geometrically equivalent to the corresponding elements of the second.

We'll explore different understandings of these definitions and their significance in due course. ${ }^{17}$ For now, note that the account seems to be on the right track. When we think about a paradigm background dependent theory (such as a special relativistic theory, i.e. one that employs a flat Minkowski manifold) we think of spacetime as a stage which remains unchanged as matter moves about, and one natural way to develop this idea is a modal gloss where spacetime doesn't change across the theory's space of nomic possibilities irrespective of how matter moves around. Moreover, some standard general relativistic

tions that are asymptotically flat at spatial infinity. As far as I'm aware nobody has extended these results to the space of all solutions, so officially the claims in the main text should be restricted to these sectors of GR.

${ }^{16}$ Here my terminology diverges from Belot's. I find my usage more natural, and it makes the ensuing discussion easier to present. Belot uses these terms differently because of his views on symmetries and asymptotic boundary conditions, which are largely orthogonal to the points I want to make here. For discussion see Belot $(2008,2017)$.

${ }^{17}$ I've given only Belot's central definitions of "full" background dependence and independence. He also defines some intermediate cases of "near" background dependence and independence but I won't discuss these here. 
theories turn out background independent on the account. For example, Belot's paradigm background independent theory with matter is general relativity coupled to dust: here the stress-energy-momentum tensor fixes a unique configuration of the dust particles (see corollary 3.14.2 in Sachs \& Wu (1977)), and so any changes to the dust that generate non-gauge equivalent solutions will correlate with changes to non-geometrically equivalent spacetime geometries via the Einstein equation.

\subsection{Looking Ahead}

My plan in the rest of the paper is to investigate what worlds must be like in order to be truly described by theories satisfying these accounts of background independence, and then to use these results to argue that none of the accounts is a non-empirical virtue. Of course I haven't even attempted to discuss every extant account of background independence, though the ones we've seen are among the most prominent, and the general morals I draw about the debate apply irrespective of one's preferred account. ${ }^{18}$

Because Belot defines a graded notion, his accounts of background dependence and independence aren't mutually exhaustive. Thus in addition to asking whether his account of background independence is a non-empirical virtue, we must also ask whether given his account the property of not being background dependent is a non-empirical virtue. This latter question is just as important as the former in terms of evaluating whether Belot's account can vindicate the pronouncements about the utility of background independence in our quest for a final physical theory (which include claims to the effect that perturbative string theories are problematic because of their background dependence).

We'll start with this latter question for Belot's account (which we'll see subsumes our investigation of the account in terms of absolute objects), then turn to diffeomorphism invariance, and then finally to Belot's account of background independence. My strategy in each case will be to first look at different conceptions of what a world might be like so as to be truly described by a theory satisfying the account, and then argue that for each of these conceptions we have little non-empirical reason to be more confident that our world is like that rather than some other way.

One final point before moving on. I'll assume throughout that nomic possibility is just metaphysical compossibility with the laws of nature, and hence that metaphysical necessity implies nomic necessity, which simplifies the discussion in what follows. This assumption is neutral between the standard view that the space of nomic possibilities is properly included in the space of metaphysical possibilities, and its main alternative where these two spaces

\footnotetext{
${ }^{18}$ Some notable omissions from my discussion are the accounts offered by Earman (2006a,b), Pooley (2017), de Haro (2015), and de Haro et al. (2016). I think each of these accounts faces problems, but I leave these as topics for another time.
} 
are identical.

\section{What's Wrong with Necessary Geometric Structure?}

Recall that for Belot background dependent theories are those with no geometrical degrees of freedom, i.e. geometric structure is nomically necessary according to the theory. Let's look at two general options for understanding this property, and for each option ask whether not being background dependent according to Belot's account is a non-empirical virtue. Afterwards, I'll explain why our discussion of this issue subsumes the question of whether containing no absolute objects is a non-empirical virtue. (Moving forward I'll sometimes use 'background dependence' and 'background independence' to describe Belot's accounts, rather than the general notions, when no unclarity will result.)

On the first understanding of the property, theories with no geometrical degrees of freedom (those that are background dependent for Belot) say only that qualitative geometric structure is nomically necessary (i.e. that all qualitative propositions about geometric structure are either nomically necessary or nomically impossible). Are there any non-empirical reasons to in general be less confident in theories with this feature than in those without it?

I don't think so. Here are two ways a world might be so as to be truly described by a background dependent theory on this understanding:

1. Assume a non-Humean conception of laws (e.g. the view that there's a fundamental 'it's a law that' operator, defended by Maudlin (2007)). The world includes a nonHumean law that spacetime has such-and-such complete qualitative geometric structure. For instance, a non-Humean law that spacetime is Minkowskian.

2. Assume a Humean best system conception of laws, which says roughly that the laws at a world are the simplest and most informative summaries of the distribution of fundamental properties and relations at that world (see Lewis (1994) and Loewer $(1996,2012)$ for details). The distribution of fundamental properties and relations in the world ensures that the simplest and most informative summary includes a claim that the world has such-and-such complete qualitative geometric structure. For example, perhaps the best Humean summary of the distribution fundamental properties and relations at a reasonably rich relationist world consistent with Special Relativity (and without tachyons) will include the claims that (i) there is a four-dimensional Minkowski spacetime in which everything is located, (ii) massive particles travel along only time-like trajectories, and (iii) photons travel along only light-like trajectories. ${ }^{19}$

\footnotetext{
${ }^{19}$ This example is a relativistic analogue of Huggett's "regularity relationism" (2006), a generalization of the standard Humean conception of laws that allows claims about a world's geometrical structure to be amongst
} 
I doubt one could argue that we ought to be less confident that our world is analogous to those described in 1 and 2 than that it isn't without appealing to empirical considerations (e.g. empirical evidence supporting GR, with its variable geometries), which our definition of being a non-empirical virtue excludes. To do so, one would need non-empirical considerations that militate against physical theories with laws stating that our world has a certain complete qualitative geometric structure (i.e. theories with laws like that spacetime is Minkowskian), and I'm not sure what these considerations could be: theories with such laws aren't in general less simple, or less elegant, don't have less unificatory or explanatory power, and so on, than theories with less stringent constraints on qualitative geometric structure. Of course there may be apriori arguments against particular background dependent theories (such as those with laws that ascribe some horribly complex complete qualitative geometric structure to the world), but what we're after are non-empirical reasons to be generally less confident in background dependent theories than their alternatives.

Let me clarify one aspect of the present dialectic. What we're looking for are not reasons to lower our confidence in background dependent visions like 1 and 2 simpliciter, but rather reasons to be less confident in them than non-background dependent visions. And crucially, the kinds of fundamental features of the worlds in 1 and 2 (e.g. the non-Humean laws of world 1) can just as easily appear in worlds truly described by non-background dependent theories. For instance, we can conceive of a world where there are non-Humean laws but the laws don't settle every qualitative question about geometric structure, and hence the world is truly described by a theory that's not background dependent on the current understanding. As a result, it wouldn't bear on the present dialectic if proponents of non-background dependence could argue on apriori grounds that some fundamental feature of world 1 or 2 (e.g. the notion of a non-Humean law) is somehow objectionable: this sort of argument wouldn't help tease non-background dependent theories apart from background dependent ones. A similar criticism would apply to arguments against world 1 on the grounds that its non-Humean laws flout some apriori norm of ideological parsimony (e.g. the norm endorsed by Sider $(2011,2013)$ and Cowling (2012)).

Let's turn now to the second option for understanding what being background dependent according to Belot's account consists in. On this option, theories with no geometrical degrees of freedom (those that are background dependent for Belot) say that all geometric structure, whether qualitative or not, is nomically necessary (i.e. that all propositions about geometric structure are either nomically necessary or nomically impossible). Here background dependent theories ascribe not only some complete qualitative geometric structure to the world, but also ascribe the complete non-qualitative geometric structure that says of

the laws in the world's best system, rather than directly encoded in the world's fundamental properties and relations. For details and critical discussion see Belot (2011b, ch3) and Stevens (2017). 
each particular spacetime point that it stands in such-and-such geometric relations to soand-so other spacetime points. Are there non-empirical reasons to be less confident in background dependent theories than non-background dependent ones on this understanding of what background dependence amounts to?

Here I think there's more to say. The first thing to note is that the analogues of worlds 1 and 2 above-which would be worlds where the complete geometric structure is a distinctively nomic necessity, deriving from a law rather than a general constraint on metaphysical modality-are non-starters. In order to secure background dependence on the present nonqualitative understanding, the laws would have to ensure that each spacetime point stands in the geometric relations it in fact stands in to the continuum-many particular spacetime points it in fact stands in those relations to. No such non-qualitative truth would be a candidate for the Humean best system, given standard conceptions of the relevant language for stating Humean laws, as well as the standard simplicity requirement on what makes some systematization the best one. And non-Humeans still accept the relevance of simplicity when determining what the laws are, and so would similarly be loathe to accept this sort of infinitary non-qualitative truth as a law. Note that trying to use quantification to bypass naming each spacetime point will secure the nomic necessity of only qualitative geometric structure, which won't take us all the way to background dependence on the present understanding. Putting the point more generally, provided only qualitative propositions can be laws of nature (which is often taken for granted, see for example Fine $(2002,261)$ ), and given also the assumption above that nomic possibility is just metaphysical compossibility with the laws of nature, there's no hope of securing background dependence as a distinctively nomic modal constraint on the present non-qualitative understanding of what background dependence amounts to.

One picture that predicts the complete non-qualitative geometric structure of the world to be nomically necessary appeals to an underlying essentialist doctrine. Here we grant the intelligibility of Fine's (1994) notion of essence, which isn't to be reduced to some more fundamental modal notions. We then assume (i) Fine's (1994) reduction of metaphysical modality to essence, which says that a proposition is metaphysically necessary in virtue of that proposition being essential to some object or plurality of objects, (ii) Maudlin's (1988; 1990) metric essentialism, which we'll understand as the doctrine that, for any spacetime point, it's essential to that point that if it's a spacetime point then it stands in the geometric relations it in fact stands in to the particular points it in fact stands in those relations to, and

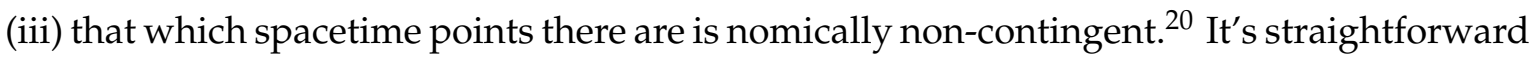

\footnotetext{
${ }^{20}$ See Teitel (Manuscript) for further discussion of these three assumptions and essentialist doctrines about spacetime more generally. Note an analogue of this package of views could be stated in purely modal terms, which is relevant for those who are skeptical of Fine's hyperintensional notion of essence. Similar points would still apply, but I'll discuss only the essence-theoretic option in the main text.
} 
to see that a world where (i), (ii), and (iii) obtain, combined with the assumption above that metaphysical necessity implies nomic necessity, will be a world where the complete (i.e. qualitative and non-qualitative) structure of spacetime is nomically necessary. Hence such worlds can be truly described only by theories that are background dependent given the present understanding.

Is there any non-empirical reason to be less confident that our world satisfies such a background dependent vision than that it doesn't? This question turns on whether there's an apriori argument against any of assumptions (i), (ii), or (iii) from the previous paragraph. I won't survey the options here, but I think there's a case to be made against each of these assumptions, and I've made such arguments in other work. ${ }^{21}$ If any of these arguments succeed then they would cast doubt on one promising means of explaining the strong haecceitistic modal constraints that background dependent theories on the present understanding ascribe to the world.

So perhaps on the second understanding of Belot's account of background dependence we can explain why not being background dependent is a non-empirical virtue. However, I suspect the first understanding of the account-where background dependent theories ascribe only some complete qualitative geometric structure to the world with nomic necessity-will be the one that concerns most readers (given the popularity of so-called "structuralist" metaphysical visions amongst philosophers of physics), and we saw that on this understanding there's little hope of explaining why not being background dependent is a non-empirical virtue. Moreover, for anti-haecceitists the two understandings stand or fall together, simply by definition, and hence those who take themselves to have apriori reason to adopt anti-haecceitism will likely take my arguments that not being background dependent, when understood purely qualitatively, isn't a non-empirical virtue to cast doubt on the importance of the haecceitistic understanding of not being background dependent as well.

Before moving on, let me explain why my arguments in this section also suggest that the account of background independence in terms of lacking absolute objects isn't a nonempirical virtue. First note that having no geometrical degrees of freedom (i.e. being background dependent for Belot) arguably implies having an absolute object. Blocking this implication would require a conception of geometrical equivalence where the elements of solutions that represent geometrical structure can be geometrically equivalent yet nonetheless differ in some respect that can't be induced by a global diffeomorphism. For example, one such conception allows that even solutions to a general relativistic theory that differ

\footnotetext{
${ }^{21}$ See Teitel (Forthcoming) for an attempt to argue that those who believe that possibly it's contingent what there is must reject Fine's reduction, i.e. assumption (i). See Teitel (Manuscript) for arguments against assumption (ii) in the setting of GR, and also arguments against the combination of assumption (iii) with standard varieties of metric essentialism (on the grounds that this combination has the implausible consequence that most propositions about the concrete world turn out nomically necessary at worlds with laws like those of GR).
} 
over the global topology of spacetime can contain geometrically equivalent metrics. I'll set such conceptions aside here: given standard representational conventions they contradict both standard haecceitist views (which would take even diffeomorphisms to induce non-geometrically equivalent geometries) and standard anti-haecceitist views (because differences in features like the global topology of spacetime are qualitative differences).

So being background dependent for Belot implies having an absolute object; and so if a theory is background independent in the sense of containing no absolute objects then it's not background dependent for Belot. To argue for an equivalence here, we'd also need to show that theories that aren't background dependent for Belot contain no absolute objects. This implication isn't plausible: not being background dependent for Belot concerns only the elements of a theory's solutions that the geometrization says represent geometrical structure, and so on its own tells us nothing about the other objects in the theory's solutions. However, for those who adopt an anti-haecceitist inspired conception of geometrical equivalence (and so don't take diffeomorphisms to generate non-geometrically equivalent geometries), the implication can be defended given a weaker account that equates background independence with lacking an absolute object that represents complete geometrical structure. ${ }^{22}$ This weaker account arguably captures the motivations behind accounts of background independence in terms of lacking absolute objects, and still allows us to defend the implication from being background independent to not being background dependent for Belot in the way we did one paragraph back. Anti-haecceitism is standardly taken on by philosophers of physics, so for most readers this weaker account of background independence in terms of absolute objects will be equivalent to the property of not being background dependent for Belot. As a result, for most readers my argument in this section that the latter property isn't a non-empirical virtue suffices to show that the former property isn't one either.

\section{Why be Diffeomorphism Invariant?}

Let's turn now to accounts that equate background independence with diffeomorphism invariance. The issues here change depending on our underlying metaphysics of modality.

\footnotetext{
${ }^{22}$ The 'complete' qualifier is needed to accommodate theories with multiple elements in their solutions that each represent different aspects of geometrical structure: such theories could have geometrical degrees of freedom despite some of the elements that represent aspects of geometrical structure being absolute objects. For example, solutions of Newton-Cartan theory as standardly presented (e.g. as in Friedman (1983, ch3.4)) contain a spatial and temporal metric that are absolute objects, yet the theory has geometrical degrees of freedom stemming from its dynamical affine connection, which varies across solutions. This theory isn't a counterexample to the implication I'm defending in the main text-from having geometrical degrees of freedom (i.e. not being background dependent for Belot) to lacking an absolute object that represents complete geometrical structure-because the spatial and temporal metrics on their own represent only certain aspects of geometrical structure. The complete geometrical structure in solutions of Newton-Cartan theory is represented only by triples consisting of the two metrics and the connection, and these triples are not absolute objects.
} 
Let's consider the various options in turn.

We'll start with a strong haecceitist package of views that implies a clear story of what the mathematical property of diffeomorphism invariance is being used to represent. According to this package of views, there are no restrictions on which spacetime points there are or on which spacetime points can play which qualitative roles that stem from some constraint on metaphysical modality (whether arising from essence or some other source), and we use diffeomorphically related solutions to represent distinct nomic possibilities, with each such solution representing only one possibility. Here diffeomorphism invariance amounts to a plenitude principle, ensuring the space of nomic possibilities is rich and doesn't contain any gaps. This lack of gaps has two facets. Suppose we use some solution $\left\langle M, O_{1}, O_{2}, \ldots\right\rangle$ of some field theory to represent the actual world. Then diffeomorphism invariance on this view ensures that there's a nomic possibility corresponding to every way of smoothly permuting the points in spacetime (represented by $M$ ), but where everything is qualitatively just as it actually is. Moreover, notice that our definition of diffeomorphism invariance from section 3.2 allows a theory's solutions to contain different four-dimensional differentiable manifolds (remember that some such manifold can consist of any objects whatsoever). This way we didn't prejudge the issue of whether which spacetime points there are is nomically non-contingent given standard representational conventions. Some may take the choice of a manifold to be a mere gauge difference between solutions, and hence regard which spacetime points there are as a nomically necessary matter. However, our definition leaves open that it's nomically contingent which spacetime points there are given standard representational conventions, i.e. that even solutions differing merely over a choice of manifold are used to represent distinct nomic possibilities. As a result, on the present haecceitist package of views, diffeomorphism invariance also amounts to ruling out gaps in modal space by ensuring that there are new nomic possibilities corresponding to every way of substituting a spacetime point with something that isn't a spacetime point but merely could be one.

Given this package of views, it's easy to explain why diffeomorphism invariance is a non-empirical virtue. Theories that fail to be diffeomorphism invariant, on the present interpretation of this property, describe worlds with arbitrary gaps in their accessible space of nomic possibilities, allowing some but not all merely haecceitistic differences in spacetime points to induce new nomic possibilities. (Note the non-arbitrary option of claiming that no merely haecceitistic differences in spacetime points generate new nomic possibilities corresponds to the anti-haecceitist option we'll look at shortly.) I'm not sure what could motivate gaps of this sort; what differences between spacetime points could make it nomically possible for some but not others to swap qualitative roles, or nomically possible for some but not others to fail to be spacetime points? ${ }^{23}$ So I think those with the present package of

\footnotetext{
${ }^{23}$ One exception here would be the view defended by Belot $(2008,2017)$, which says that we use diffeomor-
} 
modal and representational commitments, and who also equate background independence with diffeomorphism invariance, can justifiably regard background independence as a nonempirical virtue. However, this result will be of little solace to most readers, given that most philosophers of physics I'm aware of reject the present strong haecceitist package of views.

Let's turn now to anti-haecceitism (recall that this is the doctrine that metaphysical possibilities don't differ merely haecceitistically). The issue here is that anti-haecceitists generally don't use diffeomorphically related solutions to represent distinct nomic possibilities. So given these theorists' representational conventions, diffeomorphism invariance is a mathematical property with no clear correlate in their underlying metaphysics: given a diffeomorphism invariant theory, these theorists could arbitrarily select a single solution from each equivalence class of diffeomorphically related solutions, put these together to form a non-diffeomorphism invariant theory, and the result is equally well suited to represent the space of nomic possibilities as these anti-haecceitists conceive it given their representational conventions. True anti-haecceitists would need to be cautious when interpreting the nondiffeomorphism invariant theory. For instance, they may need to specify that certain properties of the objects that make up the manifolds in the selected solutions aren't physically significant; after all these could be any objects whatsoever, whether lions, tigers, bears, numbers, or spacetime points. However, this wouldn't render the non-diffeomorphism invariant theory inherently less suited to accurately represent the space of nomic possibilities as conceived by anti-haecceitists than the theory that keeps the full equivalence classes of diffeomorphically related solutions given how the anti-haecceitist standardly interprets such equivalence classes. ${ }^{24}$ As a result, diffeomorphism invariance has no clear propositional content for proponents of anti-haecceitist visions of modal reality. And so I'm not sure how such theorists might even attempt to argue that accounts of background independence in terms of diffeomorphism invariance are non-empirical virtues. ${ }^{25}$

phisms to represent differences that generate new nomic possibilities only when the diffeomorphisms don't preserve asymptotic boundary conditions. I won't discuss Belot's proposal here, though if successful it suggests that perhaps even haecceitists have little to say in favor of diffeomorphism invariant theories over their alternatives.

${ }^{24}$ Exactly which representational conventions the anti-haecceitist who employs the standard diffeomorphism invariant option should adopt will vary depending on her background metaphysical commitments, so the discussion in the main text is glossing over various decision-points. Many anti-haecceitists will espouse the convention that only what's invariant across all solutions in each diffeomorphism orbit represents fundamental structure. But they'll also all need a convention stating precisely how to interpret the points of the manifolds in the theory's solutions, such as one stating that there are no individuals corresponding to the points in the manifolds, or perhaps stating that the identities of such individuals are in some sense indeterminate (see Russell (2016) for relevant discussion).

${ }^{25}$ I should flag that the arguments in this section generalize to analogues of diffeomorphism invariance one might propose as accounts of background independence in the setting of the canonical rather than covariant formulation of GR, provided such analogues are purely mathematical constraints (such as 3D diffeomorphism invariance). Thanks to an anonymous referee here. 


\section{Why Think Everything is Necessitated by Geometry?}

Let's turn finally to Belot's account of background independence. Recall that for Belot, a theory is background independent just in case all of its physical degrees of freedom correspond to geometrical degrees of freedom. The key expression here is "corresponds": on Belot's definition, so long as every difference between nomic possibilities correlates with a difference in spacetime geometry we have background independence, irrespective of why these modal correlations obtain. We'll examine a few ways a world might be so as to predict these correlations and thereby be truly described by such background independent theories. We'll also see that, although some strategies are more promising than others, we have little reason to think that Belot's account is a non-empirical virtue.

Start with the most face-value reading of the account: background independent theories could describe worlds whose laws imply certain correlations between differences in anything there is and differences in geometry. Already we can see that this tack is hopeless if we allow merely haecceitistic differences to qualify as geometrical and physical degrees of freedom. For reasons we saw when discussing the analogous tack to secure nonqualitative background dependence in section 4, neither Humeans nor non-Humeans will think a world's laws could impose such purely haecceitistic constraints, and, more generally, plausibly only qualitative propositions can be laws of nature. For example, suppose permuting some matter but leaving everything qualitatively unchanged generates a distinct nomic possibility. Then our world's laws would need to ensure that some difference in geometry always correlates with such permutations. The natural corresponding geometrical difference here would be some merely haecceitistic difference in geometry. However, the only path I can see to securing this haecceitistic modal correlation is via a reductive doctrine where matter is somehow grounded in geometry. We'll look at such a reductive option shortly, but it goes beyond the present option where we want the requisite correlations across nomic possibilities to stem from the world's laws.

Turning to anti-haecceitist visions, here's where laws could imply the modal correlations that Belot requires for background independence. For example, in the case of GR coupled to dust, the paradigm background independent theory with matter we saw above, given anti-haecceitism these correlations are purely qualitative and implied by the Einstein equation. We can geometrize gravity away in GR because it affects all matter indiscriminately, rather than affecting matter in different ways depending on which other conditions obtain (contrast the electromagnetic force, which affects particles in different ways depending on their charges). ${ }^{26}$ Thus, in the abstract, the property of worlds that allows them to have laws (whether Humean or non-Humean) that imply the requisite qualitative correlations across

\footnotetext{
${ }^{26}$ See Friedman $(1983,195-199)$ for discussion about why gravity is special in this regard. This question is often discussed in the literature in the context of finding a precise version of Einstein's equivalence principle.
} 
nomic possibilities between all phenomena and geometry is the following: the world contains only fundamental forces like gravity, whose effects can somehow be encoded in the geometry of spacetime. ${ }^{27}$ The laws of such worlds could then amount to purely qualitative correlations between geometry and matter, which would secure background independence for Belot given anti-haecceitism.

The trouble is that it's hard to see what apriori considerations should make us more confident in worlds containing only fundamental forces that allow this kind of geometrization strategy than in those that contain some fundamental forces like electromagnetism that are sensitive to some further property and hence discriminate between different kinds of matter (again, still setting aside visions where matter is somehow grounded in geometry, which I'll consider shortly). The only considerations that seem relevant here are empirical ones, but these don't bear on the question of non-empirical virtues. Given some fundamental force that allows for the possibility of being geometrized away, there's a familiar symmetry argument supporting theories that adopt the geometrization strategy over standard alternatives. ${ }^{28}$ But what we need here are non-empirical arguments to be more confident that our world contains only fundamental forces that allow this kind of geometrization strategy, not just non-empirical arguments for being more confident in geometrized accounts of such forces over their alternatives.

So let's turn to the reductive option for securing the modal correlations that Belot requires for background independence, which I suspect is the only route to arguing that Belot's account is a non-empirical virtue. My claim is that a certain ambitious sort of supersubstantivalist metaphysics (which I'll define shortly) can obtain only at worlds that are truly described by theories that are background independent for Belot. So Belot may try to appeal to considerations in favor of this metaphysical vision in order to explain why his property is a non-empirical virtue. Let me expand on these claims, and then evaluate the prospects of this strategy.

I'll take supersubstantivalism to be the view that there is no fundamental occupation or location relation (usually taken to relate matter and spacetime points or regions). This characterization intentionally leaves open lots of what has gone under the 'supersubstantivalist' label in the literature: e.g. conceptions of reality where matter (including fields) is identical to spacetime regions, or properties of spacetime regions, or somehow grounded in spacetime regions, and the options go on. (See Lehmkuhl (2016) for discussion of some of the decision-points here, and Schaffer (2009) for quite general arguments in favor of the view.)

\footnotetext{
${ }^{27}$ Of course, at worlds where gravity is geometrized away there is no fundamental gravitational force. I'll still speak of "fundamental forces" being geometrized away, though for this reason this locution isn't strictly speaking accurate.

${ }^{28}$ For example, the symmetry argument for moving from potential-based formulations of Newtonian gravitational theories to Newton-Cartan theory, discussed by Friedman (1983, ch3.4) and Knox (2014). See Dasgupta (2016) for general discussion about how to understand symmetry arguments.
} 
Still the characterization rules out the traditional picture where you have matter, spacetime, and the former standing in a fundamental occupation relation to the latter.

The modest form of supersubstantivalism still countenances all of the familiar fundamental properties of matter like charge and spin, but holds that these fundamental properties are instantiated by spacetime points or regions, or some neutral plenum. This less ambitious form of the view has features to recommend it (see again the arguments in Schaffer (2009)). However, this sort of view is available even in a non-relativistic setting, and doesn't rely on any features peculiar to GR. And as a result, it's unsurprising that this form of supersubstantivalism has little to do with Belot's criterion for background independence (indeed even theories that are background dependent for Belot leave open this modest sort of supersubstantivalism). The more ambitious brand of supersubstantivalism tries to do without the fundamental properties traditionally associated with matter, and instead holds that these properties are somehow reducible to fundamental properties traditionally associated with spacetime (curvature, topology, smoothness, etc.). ${ }^{29}$ An example of this sort of geometrize-everything project was Wheeler's (1962) program of geometrodynamics, which took GR to inspire just this kind of ambitious supersubstantivalism (see Earman (1972) and Stachel (1972) for discussion of why this program was abandoned).

My definition of the ambitious project was intentionally vague, claiming that the fundamental properties traditionally associated with matter are "somehow reducible" to the fundamental properties traditionally associated with spacetime. But one can argue that however we cash out this ideology, ambitious supersubstantivalism can be true only at worlds where the modal correlations that Belot requires for background independence obtain, on either the haecceitist or anti-haecceitist conceptions of such correlations that we've looked at. The way to see this is to look at the contrapositive. If the modal correlations fail then there are distinct nomic possibilities that agree on the properties of spacetime geometry but disagree on the properties of matter. Given anti-haecceitism, this amounts to nomic possibilities that differ over the qualitative properties of matter but don't differ over the properties of spacetime geometry. Given haecceitism, we either have an analogous situation, or else nomic possibilities where matter differs merely haecceitistically without any corresponding difference in spacetime geometry. To see how either of these commitments conflicts with ambitious supersubstantivalism, we need to suppose only that the sense of reduction the ambitious supersubstantivalist intends implies necessitation, i.e. whatever she means by claiming that the fundamental properties traditionally associated with matter are reducible to the fundamental properties traditionally associated with spacetime, she thinks the implication from propositions about the latter to propositions about the former holds

\footnotetext{
${ }^{29}$ See Sklar $(1974,166,222-223)$ and Lehmkuhl (2016) for related distinctions between more and less ambitious versions of supersubstantivalism.
} 
with metaphysical necessity (and hence nomic necessity, given the assumption in section 3.5). Given this necessitation supposition, for both anti-haecceitists and haecceitists there's no hope of implementing the ambitious project if Belot's modal correlations fail: fix the fundamental geometric properties and there are still distinct nomic possibilities for what matter is like (whether differing qualitatively or merely haecceitistically). There's room to resist the crucial claim in this reasoning linking reduction and necessitation on certain conceptions of reduction (see for instance Skiles (2015)), however I'm content to grant Belot this claim here.

Does this result - that ambitious supersubstantivalism can be true only at worlds where the modal correlations that Belot requires for background independence obtain-show that background independence on Belot's account is a non-empirical virtue? Here's why I'm skeptical.

There are various apriori arguments for ambitious supersubstantivalism. First, ambitious supersubstantivalists needn't regard any of the traditional properties of matter as fundamental, instead reducing these to fundamental geometric properties. So one can argue for the view using considerations of ideological parsimony, given that we granted in the introduction that apriori there's an epistemic norm in favor of simplicity. Second, the view eliminates the need for a fundamental occupation or location relation, which further contributes to ideological parsimony, and can perhaps be motivated by independent apriori arguments against conceptions of reality with non-symmetric relations (of the sort offered by Dorr (2004)). Finally, arguably ambitious supersubstantivalism enjoys other traditional non-empirical virtues, such as elegance or unification, however these are made precise.

Putting these points together, one can argue that when comparing a theory that's background independent on Belot's account to a rival non-background independent theory, we ceteris paribus ought to be at least somewhat more confident in the former than in the latter for apriori reasons, because only the background independent theory leaves open the possibility of ambitious supersubstantivalism. My worry is that at best this gives background independent theories a small boost, but not nearly enough to vindicate the uses to which background independence has been put by theorists working on quantum gravity (i.e. on any reasonable resolution of the vagueness of the "to some non-trivial degree" qualifier as it appears in our definition of a non-empirical virtue, the fact that background independent theories leave open ambitious supersubstantivalism doesn't license being more confident in them than in their rivals to some non-trivial degree). Put another way, even though all conceivable ambitious supersubstantivalist worlds can be truly described only by theories that are background independent for Belot, we nonetheless ought to distribute the vast majority of our priors in background independent theories over worlds where those theories are true but ambitious supersubstantivalism fails. Some may be more optimistic about ambitious supersubstantivalism than I am (e.g. Lehmkuhl (2016)), though I suspect most will share 
my verdicts here.

The upshot of our discussion in this section is that Belot's account of background independence isn't a non-empirical virtue, on any option for understanding what the account is used to say about the world.

\section{Conclusion}

I'll close by summarizing my results and stating a general moral.

My first goal was to investigate the metaphysical question of what a world must be like in order to be truly described by a background independent theory given extant accounts of background independence. I extracted the following propositional content (all stated here as properties of an arbitrary theory $T$ ) from the various mathematical properties at issue in the literature, arriving at five non-mathematical notions of background independence:

Background Independence 1: Some qualitative proposition about geometric structure is nomically contingent according to $T$.

Background Independence 2: Some proposition (whether qualitative or haecceitistic) about geometric structure is nomically contingent according to $T$.

Background Independence 3: According to T, for every nomic possibility $w$, there's a distinct nomic possibility corresponding to every way of smoothly permuting some spacetime points at $w$ but leaving everything qualitatively unchanged, and also a distinct nomic possibility corresponding to every way of substituting a spacetime point at $w$ with something that's not a spacetime point but merely could be one.

Background Independence 4: No nomic possibilities according to $T$ differ qualitatively without also differing over some qualitative proposition about geometric structure.

Background Independence 5: No nomic possibilities according to $T$ differ (whether qualitatively or merely haecceitistically) without also differing over some proposition (whether qualitative or haecceitistic) about geometric structure.

I also thereby extracted five non-mathematical notions of background dependence, corresponding to the properties of not being Background Independent 1, not being Background Independent 2, and so on. In particular, the two understandings of Belot's account of background dependence that we discussed were the properties of not being Background In-

dependent 1 and not being Background Independent 2. Moreover, for each of these ten 
modal properties, we looked at different metaphysical visions in virtue of which the property might be instantiated.

My second goal was to determine whether any of these notions of background independence is a non-empirical virtue. My results here were largely negative. Starting with some good news for fans of background independence, we saw reason to think that Background Independence 2 is a non-empirical virtue. Those who take themselves to have some apriori motivation for anti-haecceitism could use this result to argue that Background Independence 1 must also be a non-empirical virtue (because Background Independence 1 and 2 must stand or fall together for anti-haecceitists), though we also saw independent reasons not to regard Background Independence 1 as a non-empirical virtue, which for antihaecceitists must also tell against Background Independence 2. In section 5 we saw that if there's apriori reason to be confident in a particular haecceitist package of views then Background Independence 3 is plausibly a non-empirical virtue, while of course anti-haecceitists by definition must believe that no theory satisfies this property. Finally, we saw in section 6 that arguably neither Background Independence 4 nor Background Independence 5 is a non-empirical virtue, irrespective of one's views on the apriori plausibility of either antihaecceitism or haecceitism (though of course for anti-haecceitists these two properties must stand or fall together).

Let me finally state a moral of our discussion for those theorizing about background independence and the metaphysics of our physical theories more generally. A large component of our discussion was distilling these five constraints on nomic modality (Background Independence 1-5) from mathematical properties that impose constraints on solution spaces (i.e. classes of mathematical models). The latter formal constraints may or may not express the former modal properties, depending on which representational decisions we adopt for how to use the mathematical formalism. In general, mathematical properties of solution spaces on their own are purely formal constraints, which express no propositional content imposing a constraint on the (non-mathematical) world, let alone imposing a constraint on nomic modality. Yet the literature largely eschews discussing modal properties like Background Independence 1-5 directly, instead taking the roundabout route of discussing only formal properties of solution spaces, and moreover often not making explicit what nonmathematical properties these formal properties are being used to represent.

I take our discussion to support the moral that we can make progress on substantive questions in the vicinity of debates over background independence and other topics in the foundations of spacetime theories by focussing more on precisely formulated constraints on nomic modality (which are often only implicitly at issue), rather than taking this standard detour through solution spaces and representational conventions for using these mathematical objects to describe the non-mathematical world. For instance, once I managed to distill 
Background Independence 1-5 from the mathematical properties standardly discussed in their stead, the question of whether any of these is a non-empirical virtue was reasonably straightforward to adjudicate. We shouldn't forget a common lesson drawn from the older discussion of general covariance (recall section 2.1), which I mentioned in the introduction: candidates for properties of a physical theory that ought to make us more confident that the theory is true should pertain to what the theory says about the world, i.e. its propositional content, not the choice of formalism used to express that theory. To give another example where unwillingness to discuss the modal properties at issue directly has obscured the debate, it's uncontroversial that the anti-haecceitist must believe that no theories satisfy Background Independence 3, simply by definition given her modal doctrine, and hence that she ought not take this property to be a non-empirical virtue. Yet we saw in section 5 that there's no straightforward way to map this anti-haecceitist modal commitment onto the mathematical property of being diffeomorphism invariant, which is the purely formal analogue of Background Independence 3 standardly at issue in the discussion: neither diffeomorphism invariant nor non-diffeomorphism invariant mathematical objects (e.g. solution spaces) are inherently more suited to accurately represent the space of nomic possibilities as the anti-haecceitist conceives it given the representational conventions she usually adopts. In general, then, while of course mathematical developments have been and will continue to be integral to the progress of physics (and science more generally), we should never lose

sight of the fact that our ultimate interest is the non-mathematical world we're attempting to use that mathematics to describe.

\section{Acknowledgements}

Thanks most of all to Cian Dorr, for extremely helpful comments and encouragement at every stage of the paper's development. I'm also particularly grateful to David Albert, Gordon Belot, Ben Holguín, Anja Jauernig, Tim Maudlin, and the two anonymous referees for extensive comments that significantly improved the paper. Finally, for helpful comments and discussion about this material, thanks to Thomas Barrett, Kevin Coffey, Hartry Field, Marko Malink, Tushar Menon, James Read, Michael Strevens, Daniel Sudarsky, and David Wallace. 


\section{References}

Anderson, James L. 1967. Principles of Relativity Physics. Academic Press.

Andersson, Lars. 1987. Momenta and reduction for general relativity. Journal of Geometry and Physics 4. $289-314$.

Baez, John C. 2001. Higher-Dimensional Algebra and Planck Scale Physics. In Craig Callender \& Nick Huggett (eds.), Physics Meets Philosophy at the Planck Scale, 177-195. Cambridge University Press.

Belot, Gordon. 2008. An elementary notion of gauge equivalence. General Relativity and Gravitation 40(1). 199215.

Belot, Gordon. 2011a. Background-independence. General Relativity and Gravitation 43(10). 2865-2884.

Belot, Gordon. 2011b. Geometric Possibility. Oxford ; New York: Oxford University Press.

Belot, Gordon. 2017. Fifty Million Elvis Fans Can't be Wrong. Noûs .

Bennett, Karen \& Brian McLaughlin. 2014. Supervenience. In Edward N. Zalta (ed.), The Stanford Encyclopedia of Philosophy, Metaphysics Research Lab, Stanford University spring 2014 edn.

Brighouse, Carolyn. 1994. Spacetime and Holes. PSA: Proceedings of the Biennial Meeting of the Philosophy of Science Association 1. 117-125.

Cowling, Sam. 2012. Ideological parsimony. Synthese 190(17). 3889-3908.

Dasgupta, Shamik. 2016. Symmetry as an Epistemic Notion (Twice Over). The British Journal for the Philosophy of Science 67(3). 837-878.

Dorr, Cian. 2004. Non-Symmetric Relations. Oxford Studies in Metaphysics 1. 155-92.

Earman, John. 1972. Some Aspects of General Relativity and Geometrodynamics. The Journal of Philosophy 69(19). 634-647.

Earman, John. 1986. A Primer on Determinism. D. Reidel.

Earman, John. 2006a. The Implications of General Covariance for the Ontology and Ideology of Spacetime. In Dennis Dieks (ed.), Philosophy and Foundations of Physics, vol. 1 The Ontology of Spacetime, 3-23. Elsevier.

Earman, John. 2006b. Two Challenges to the Requirement of Substantive General Covariance. Synthese 148(2). 443-68.

Earman, John \& John Norton. 1987. What price spacetime substantivalism? The hole story. British Journal for the Philosophy of Science 515-525.

Field, Hartry. 1984. Can We Dispense with Space-Time? PSA:Proceedings of the Biennial Meeting of the Philosophy of Science Association 2. 33-90. Citations to reprinted version in Field 1989, Realism, Mathematics, and Modality, 171-226. Oxford: Basil Blackwell.

Fine, Kit. 1994. Essence and Modality. Philosophical Perspectives 8. 1-16.

Fine, Kit. 2002. The Varieties of Necessity. In Tamar Szabo Gendler \& John Hawthorne (eds.), Conceivability and Possibility, 253-281. Oxford University Press.

Friedman, Michael. 1983. Foundations of Space-Time Theories: Relativistic Physics and Philosophy of Science. Princeton: Princeton University Press.

Giulini, Domenico. 2007. Some Remarks on the Notions of General Covariance and Background Independence. Lecture Notes in Physics 721. 105-20. 
de Haro, Sebastian. 2015. Dualities and emergent gravity: Gauge/gravity duality. Studies in History and Philosophy of Modern Physics .

de Haro, Sebastian, Daniel R. Mayerson \& Jeremy N. Butterfield. 2016. Conceptual Aspects of Gauge/Gravity Duality. Foundations of Physics 46(11). 1381-1425.

Hoefer, Carl. 1996. The Metaphysics of Space-Time Substantivalism. The Journal of Philosophy 93(1). 5.

Huggett, Nick. 2006. The Regularity Account of Relational Spacetime. Mind 115(457). 41-73.

Isenberg, J. A. \& J. E. Marsden. 1982. A slice theorem for the space of solutions of Einstein's equations. Physics Reports 89. 179-222.

Kaplan, David. 1975. How to Russell a Frege-Church. The Journal of Philosophy 72(19). 716-729.

Knox, Eleanor. 2014. Newtonian Spacetime Structure in Light of the Equivalence Principle. The British Journal for the Philosophy of Science 65(4). 863-880.

Lehmkuhl, Dennis. 2016. The Metaphysics of Super-Substantivalism. Noûs .

Lewis, David. 1994. Humean Supervenience Debugged. Mind 103(412). 473-490.

Loewer, Barry. 1996. Humean Supervenience. Philosophical Topics 24(1). 101-127.

Loewer, Barry. 2012. Two accounts of laws and time. Philosophical Studies 160(1). 115-137.

Maudlin, Tim. 1988. The Essence of Space-Time. PSA: Proceedings of the Biennial Meeting of the Philosophy of Science Association 1988. 82-91.

Maudlin, Tim. 1990. Substances and space-time: What Aristotle would have said to Einstein. Studies in History and Philosophy of Science Part A 21(4). 531-561.

Maudlin, Tim. 2007. A Modest Proposal Concerning Laws, Counterfactals, and Explanations. In The Metaphysics Within Physics, 5-49. Oxford: Oxford University Press.

Melia, Joseph. 1999. Holes, Haecceitism and Two Conceptions of Determinism. The British Journal for the Philosophy of Science 50(4). 639-664.

Misner, C.W., K.S. Thorne \& J.A. Wheeler. 1973. Gravitation. San Francisco: W. H. Freeman.

Norton, John D. 1993. General Covariance and the Foundations of General Relativity: Eight Decades of Dispute. Reports of Progress in Physics 56. 791-861.

Norton, John D. 2015. The Hole Argument. In Edward N. Zalta (ed.), The Stanford Encyclopedia of Philosophy, Metaphysics Research Lab, Stanford University fall 2015 edn.

Pitts, J. B. 2006. Absolute objects and counterexamples: Jones-Geroch dust, Torretti constant curvature, tetradspinor, and scalar density. Studies in History and Philosophy of Modern Physics 37(2). 347-371.

Pooley, Oliver. 2006. Points, Particles and Structural Realism. In Dean Rickles, Steven French \& Juha Saatsi (eds.), The Structural Foundations of Quantum Gravity, 83-120. Oxford University Press.

Pooley, Oliver. 2010. Substantive General Covariance: Another Decade of Dispute. In Mauricio Suárez, Mauro Dorato \& Miklós Rédei (eds.), EPSA Philosophical Issues in the Sciences: Launch of the European Philosophy of Science Association, 197-209. Springer.

Pooley, Oliver. 2017. Background Independence, Diffeomorphism Invariance and the Meaning of Coordinates. In Dennis Lehmkuhl, Gregor Schiemann \& Erhard Scholz (eds.), Towards a Theory of Spacetime Theories (Einstein Studies 13), 105-143. Springer New York. 
Read, James. 2016. Background Independence in Classical and Quantum Gravity. B.Phil. Thesis, Oxford University.

Rickles, Dean. 2008. Who's Afraid of Background Independence? In Dennis Dieks (ed.), The Ontology of Spacetime Ii, 133-52. Elsevier.

Rovelli, Carlo. 1997. Halfway Through the Woods: Contemporary Research on Space and Time. In John Earman \& John Norton (eds.), The Cosmos of Science, 180-223. University of Pittsburgh Press.

Rovelli, Carlo. 2001. Quantum Spacetime: What Do We Know? In Craig Callender \& Nick Huggett (eds.), Physics Meets Philosophy at the Planck Scale, 101-22. Cambridge University Press.

Russell, Jeffrey Sanford. 2016. Qualitative Grounds. Philosophical Perspectives 30(1). 309-348.

Sachs, Rainer \& Hung-Hsi Wu. 1977. General Relativity for Mathematicians. Springer Science \& Business Media.

Schaffer, Jonathan. 2009. Spacetime the one substance. Philosophical Studies 145(1). 131-148.

Sider, Theodore. 2011. Writing the Book of the World. Oxford: Oxford University Press.

Sider, Theodore. 2013. Against Parthood. In Karen Bennett \& Dean Zimmerman (eds.), Oxford Studies in Metaphysics, vol. 8, 237-293. Oxford: Oxford University Press.

Skiles, Alexander. 2015. Against Grounding Necessitarianism. Erkenntnis 80(4). 717-751.

Sklar, Lawrence. 1974. Space, Time and Spacetime. University of California Press.

Skow, Bradford. 2008. Haecceitism, Anti-Haecceitism and Possible Worlds. The Philosophical Quarterly 58(230). 98-107.

Smolin, Lee. 2006a. The Case for Background Independence. In Dean Rickles, Steven French \& Juha Saatsi (eds.), The Structural Foundations of Quantum Gravity, 196-239. Oxford University Press.

Smolin, Lee. 2006b. The Trouble With Physics: The Rise of String Theory, the Fall of a Science, and What Comes Next. Boston: Houghton Mifflin Harcourt.

Sorkin, Rafael D. 2002. An example relevant to the kretschmann-einstein debate. Modern Physics Letters A 17(11). 695-700.

Stachel, John. 1972. The Rise and Fall of Geometrodynamics. PSA: Proceedings of the Biennial Meeting of the Philosophy of Science Association 1972. 31-54.

Stevens, Syman. 2017. Regularity relationalism and the constructivist project. The British Journal for the Philosophy of Science .

Teitel, Trevor. Forthcoming. Contingent Existence and the Reduction of Modality to Essence. Mind .

Teitel, Trevor. Manuscript. Holes in Spacetime: Some Neglected Essentials .

Wald, Robert M. 1984. General Relativity. Chicago: University Of Chicago Press.

Wheeler, J. A. 1962. Geometrodynamics. New York: Academic Press. 